\title{
Bacteriospermia, extended spectrum beta lactamase producing Gram-negative bacteria and other factors associated with male infertility in Mwanza, Tanzania: a need of diagnostic bacteriology for management of male infertility
}

\author{
Vitus Silago ${ }^{1}$, Yusuph Mukama ${ }^{1}$, Anna L Haule ${ }^{1}$, Frank Chacha $^{2}$, John Igenge ${ }^{2}$, \\ Martha F Mushi ${ }^{1}$, Stephen E Mshana ${ }^{1}$
}

1. Department of Microbiology and Immunology, Weill Bugando School of Medicine, Catholic University of Health and Allied Sciences, Bugando. P. O. Box 1464 Mwanza, Tanzania.

2. Department of Urology, Weill Bugando School of Medicine, Catholic University of Health and Allied Sciences, Bugando. P. O. Box 1464 Mwanza, Tanzania.

\section{Emails:}

YS:yusuphmukamaboy@gmail.com;ALH: annaluogab@gmail.com; FC: frank888990@gmail.com; JI: johnigenge@hotmail.com; MFM: mushimartha@gmail.com; SEM: stephen72mshana@gmail.com

\begin{abstract}
Background: Infections caused by Extended spectrum beta lactamase (ESBL) producing bacterial are global challenge. There is limited information on the magnitude of bacteriospermia, ESBL producing Gram-negative bacteria (GNB) causing bacteriospermia and factors associated with male infertility. This study determined magnitude of bacteriospermia, ESBL-GNB and other factors association with infertility among presumptive infertile men in Mwanza, Tanzania.

Methods: A cross-sectional hospital-based study was conducted between May 2017 and July 2018 among 137 presumptive infertile men. Semen specimens were self-collected by masturbation into clean, sterile and none-spermicidal containers and processed following laboratory standard operating procedures (SOPs). Data analysis was done using STATA 13.0.

Results: Gram-negative bacteria were predominantly isolated (86.4\%), of which $31.6 \%$ were ESBL producers. In a total 44 bacteria were isolated from semen culture. The bla ${ }_{\text {Стх-м }}$ gene was detected in $75 \%$ of phenotypically confirmed ESBL producers. Infertility was independently found to be associated with abnormal spermatozoa morphology (OR (95\%CI): 14.48(3.17-66.05)) and abnormal spermatozoa motility (OR (95\%CI): 0.05(0.01-0.24)). However, neither bacteriospermia (OR (95\%CI): 0.86(0.292.59)) nor ESBL bacteriospermia (OR (95\% CI): 0.13(0.01-1.22)) was found to be associated with infertility.

Conclusion: One third of bacteriospermia is due to ESBL-producers with history of antibiotic use being protective factor for infertility. Abnormal spermatozoa morphology and poor spermatozoa forward motility independently predicted infertility.

Keywords: bacteriospermia; blaCTX-M; male infertility; extended spectrum beta lactamase; Mwanza; Tanzania.

DOI: https://dx.doi.org/10.4314/ahs.v20i1.4

Cite as: Silago V, Mukama Y, Haule AL, Chacha F, Igenge J, Mushi MF, et al. Bacteriospermia, extended spectrum beta lactamase producing Gram-negative bacteria and other factors associated with male infertility in Mwanza, Tanzania: a need of diagnostic bacteriology for management of male infertility. Afri Health Sci. 2020;20(1):4-13. https:// dx.doi.org/10.4314/abs.v20i1.4
\end{abstract}

\section{Corresponding author:}

Vitus Silago,

Department of Microbiology and Immunology,

Weill Bugando School of Medicine, CUHAS

Mwanza, Tanzania

P. O. Box 1464

Email: vsilago.silago2@gmail.com

\section{Introduction}

Bacteria, fungi, protozoa and viruses are agents implicated in male urogenital tract and accessory sex gland infections and account for about $15 \%$ of male infertility $^{1,2}$. Bacteria like; Staphylococcus aureus, Enterococcus faecalis, Staphylococcus saprophyticus, Escherichia coli, Pseudomonas aeruginosa and Klebsiella pneumoniae are the most common pathogens reported to be isolated from semen culture

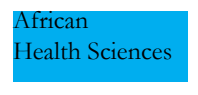

(C) 2020 Silago V et al. Licensee African Health Sciences. This is an Open Access article distributed under the terms of the Creative commons Attribution License
(https://creativecommons.org/licenses/BY/4.0), which permits unrestricted use, distribution, and reproduction in any medium, provided the original work is properly
cited. 
and causing bacteriospermia ${ }^{3,4}$. Bacteriospermia has been found to cause infertility due to various factors such as: deterioration of spermatogenesis, alteration of acrosome and sperm morphology, auto-immune processes induced by inflammation, increased sperm DNA fragmentation due to formation of reactive oxygen species and obstruction of genital tracts due to fibrosis and inflammation ${ }^{3,5}$.

Production of Extended spectrum beta lactamase (ESBL) among multi-drug resistant (MDR) Gram-negative bacteria is the common antibiotic resistance mechanism ${ }^{6}$. E. coli, K. pneumoniae, Citrobacter spp., Enterobacter spp., Acinetobacter spp. and Pseudomonas aeruginosa are common ESBL producing Gram-negative bacteria $(\mathrm{GNB})^{6,7}$. The blaCTX-M gene is documented to account over $75 \%$ of ESBL producing gram negative bacteria clinically isolated however little is known on its prevalence among ESBL producing Gram-negative bacteria causing bacteriosper$\mathrm{mia}^{8}$. Here in, we report the magnitude of bacteriospermia, blaCTX-M among ESBL producing Gram-negative bacteria and other factors associated with male infertility in Mwanza, Tanzania. This is the first study to report magnitude of bacteriospermia, ESBL producing GNB and other factors associated with male infertility from Tanzania. These data are important in the management of bacteriospermia in our setting where there is high prevalence of ESBL producing Gram-negative bacteria ${ }^{9-11}$.

\section{Methods}

\section{Study design, duration, population and setting}

This cross-sectional hospital-based study was conducted between May 2017 and July 2018 involving 137 presumptive infertile men (whose female couples were medically confirmed fertile) attending reproductive health/infertility clinics in Mwanza, Tanzania. Standardized data collection tools used to collect socio-demographic and clinical characteristics of the study participants. Semen specimens were self-collected by masturbation into wide mouth, clean, sterile and spermatozoa non-toxic specimen containers (Hunter Scientific Limited, UK) after consented voluntarily and sexual abstinence of a minimum of 3 days. Patients were instructed to pass urine and then thoroughly cleaned their hands and penis with clean water and non-antiseptic soap ${ }^{12}$. Specimens were brought to Central Pathology Laboratory, department of Histopathology at the Bugando Medical Centre (BMC) for semen analysis at room temperature within 30 minutes after collection and Catholic University of Health and Allied Sciences (CUHAS) multipurpose laboratory in cold box $\left(2-8^{\circ} \mathrm{C}\right)$ for semen culture within one hour of collection. PCR to detect blaCTX-M gene was done at National Institute of Medical Research (NIMR), Mwanza.

In this study, presumptive male infertility refers to male's inability to make fertile female partner pregnant for a period of $\geq 1$ year of active sexual practices without protections while medically confirmed infertility among men refers male infertility ${ }^{13-15}$. Male infertility can be medically confirmed by examining spermatozoa quality and quantity in an ejaculate ${ }^{13,14}$. This includes: spermatozoa concentration, morphology and/or forward motility ${ }^{13,14}$.

\section{Semen analysis, culture and identification of signifi- cant isolated bacteria}

Semen analysis involved the following parameters; colour (grey to opalescent), volume $(2-6 \mathrm{ml})$, viscosity $(<2 \mathrm{~cm}$ dropping threads from pipette), $\mathrm{pH}$ (7.2-8.2), motility, morphology and spermatozoa count (20-120 million/ milliliter) per SOPs and WHO guidelines12. Semen analysis was performed by skilled and experienced laboratory scientist ( $>5$ working years) and two other laboratory technicians ( $>3$ working years) were used to confirm for the validity of the results. Semen specimens were inoculated onto blood agar (BA) and MacConkey agar (MCA) plates followed by aerobic incubation at $37^{\circ} \mathrm{C}$ for $24-48$ hours. A pure significant growth $\left(\geq 10^{3} \mathrm{CFU} / \mathrm{ml}\right.$ growth) of bacteria were further identified to species level by inhouse biochemical identification tests; Gram stain, catalase, slide coagulase, novobiocin, bacitracin, bile esculin and optochin for Gram-positive bacteria and Gram stain, triple sugar iron (TSI), sulfur indole motility (SIM), Simmons citrate, urease and oxidase for Gram-negative bacteria $^{16}$.

\section{Antibiotic susceptibility testing}

Antibiotic susceptibility testing (AST) was performed on Muller Hinton agar (MHA) plates by Kirby-Bauer disc diffusion method as per CLSI:2010 guidelines ${ }^{17}$. Erythromycin $15 \mu \mathrm{g}$, clindamycin $2 \mu \mathrm{g}$, vancomycin $30 \mu \mathrm{g}$, gentamicin $10 \mu \mathrm{g}$, cefoxitin $30 \mu \mathrm{g}$ (for $S$. aureus only) and ciprofloxacin $5 \mu \mathrm{g}$ were used for gram positive bacteria while ampicillin $10 \mu \mathrm{g}$, sulphamethoxazole-trimethoprim 1.25/23.75 $\mu$ g, gentamicin $10 \mu \mathrm{g}$, ciprofloxacin $5 \mu \mathrm{g}$, amoxycillin-clavulanic acid 20/10 $\mathrm{g}$, ceftriaxone $30 \mu \mathrm{g}$, ceftazidime $30 \mu \mathrm{g}$, 
piperacillin-tazobactam 100/10 $\mu \mathrm{g}$ and meropenem $10 \mu \mathrm{g}$ were used for gram negative bacteria.

\section{Phenotypic detection of ESBL producing gram neg- ative bacteria}

Double disc synergy (DDS) technique was used to detect ESBL producing gram negative bacteria as reported previously17. Briefly, ceftazidime-clavulanic acid and ceftazidime plain discs were seeded on MHA plate with test organisms. The plates were incubated for 24 hours at $37^{\circ} \mathrm{C}$. The difference of zones of inhibitions of $\geq 5 \mathrm{~mm}$ between ceftazidime-clavulanic acid and ceftazidime plain was interpreted as ESBL producer ${ }^{18,19}$.

\section{Molecular characterization of blaCTX-M gene from ESBL producing gram negative bacteria}

Heat treatment technique was performed to extract bacterial DNA with minor modification from previous study ${ }^{20}$. Two colonies of fresh grown bacteria were suspended into DNase/RNase free tubes containing $500 \mu \mathrm{L}$ of sterile de-ionized water, mixed by vortexing and boiled at $100^{\circ} \mathrm{C}$ for 10 minutes. Tubes were centrifuged at 12000 $\mathrm{rpm}$ for 10 minutes to obtain $5 \mu \mathrm{L}$ of supernatant of each test bacteria for PCR.

PCR was performed for phenotypically confirmed ESBL producing gram negative bacteria to determine the presence of blaCTX-M gene as previously reported ${ }^{21}$. CTX-M3G; forward primer: 5'-GTTACAATGTGTGAGAAGCAG-3' and reverse primer: 5'-CCGT'TCCGCTATTACAAAC-3' were used. Briefly, PCR amplification was carried on thermocycler machine (GeneAmp ${ }^{\circledR}$ PCR System 9700, ThermoFishers Scientific, Singapore) as previously explained. Briefly, PCR were conditioned at; initial denaturation at $94^{\circ} \mathrm{C}$ for 5 minutes and cycles: 1 ; denaturation at $94^{\circ} \mathrm{C}$ for 60 seconds, 2 ; annealing at $55^{\circ} \mathrm{C}$ for 30 seconds and 3 ; extension at $72^{\circ} \mathrm{C}$ for 60 seconds and final extension at $72^{\circ} \mathrm{C}$ for 5 minutes. PCR products were visualized under UV illumination on gel electrophoresis by using $2 \%$ agarose gel stained with redsafe $(7.5 \mu \mathrm{L}$ of redafe were added into $150 \mathrm{ml}$ of TBE suspended with $3 \mathrm{~g}$ of agarose powder). The amplicon with band size of $1000 \mathrm{bp}$ was annotated as blaCTX-M gene, (Figure 1). E. coli ATCC 25922 was used as negative control organisms.

\section{Statistical analysis}

Data analysis was done by using STATA 13.0 version. Continuous data were presented as mean ( \pm SD) and categorical data as percentages. Logistic regression analysis was used to show association between male infertility and independent variables. A p-value of less than 0.05 at $95 \%$ confidence interval was considered as statistically significant.

\section{Ethical considerations}

Ethical clearance to conduct this study was obtained from a joint BMC/CUHAS ethics and review committee and given ethical numbers: CREC 329/2017 and updated in 2018 by certificate number $719 / 2018$. Written informed consent forms were obtained from study participants before enrollment in this study. Laboratory results; semen analysis, and culture and sensitivity were submitted to respective clinicians for patient management

\section{Results \\ Socio-demographic and clinical characteristics of study participants}

A total of 137 participants were enrolled during this study period. The mean age $( \pm S D)$ and mean infertility duration $( \pm S D)$ was $33 \pm 6.9$ years and $2.7 \pm 2$ years, respectively. The majorities of participants were living in urban areas $(64.2 \%), 54.0 \%$ had tertiary education and $97.1 \%$ enrolled from BMC. The following participants reported history of; $2.2 \%$ fever, $16.1 \%$ antibiotic use within one month prior to enrollment in this study, 18.3\% UTI and $3.7 \%$ sexually transmitted diseases (STDs). Of the 22 participants with history of antibiotic use, 13 used for $\leq 5$ days while of 25 participants with history of UTI, 7 purchased antibiotic without prescription, (Table 1). 
Table 1: Socio-demographic and clinical characteristics of study participants

\begin{tabular}{|c|c|c|c|}
\hline \multicolumn{2}{|l|}{ Variables } & Frequency (n) & Percentage (\%) \\
\hline \multicolumn{2}{|l|}{ Mean (+/- SD) age (years) } & $33(+/-6.9)$ & - \\
\hline \multicolumn{2}{|c|}{ Mean (+/-SD) infertility duration (years) } & $2.7(+/-2)$ & - \\
\hline \multirow[t]{2}{*}{ Residence $(\mathrm{N}=137)$} & Urban & 88 & 64.2 \\
\hline & Rural & 49 & 35.8 \\
\hline \multirow{4}{*}{$\begin{array}{l}\text { Level of } \\
\text { education }(N=137)\end{array}$} & Uneducated & 3 & 2.2 \\
\hline & Primary & 10 & 7.3 \\
\hline & Secondary & 50 & 36.5 \\
\hline & Tertiary & 74 & 54.0 \\
\hline \multirow{3}{*}{$\begin{array}{l}\text { Recruitment } \\
\text { clinic }(\mathrm{N}=137)\end{array}$} & Bugando Medical Centre & 133 & 97.1 \\
\hline & Kamanga Hospital & 2 & 1.4 \\
\hline & Manjis health care centre & 2 & 1.4 \\
\hline \multirow{2}{*}{$\begin{array}{l}\text { History of } \\
\text { fever }(N=137)\end{array}$} & Yes & 3 & 2.2 \\
\hline & No & 134 & 97.8 \\
\hline \multirow{2}{*}{$\begin{array}{l}\text { Previous antibiotic } \\
(\mathrm{N}=137)\end{array}$} & Yes & 22 & 16.1 \\
\hline & No & 115 & 83.9 \\
\hline \multirow{4}{*}{$\begin{array}{l}\text { Duration of antibiotic } \\
\text { use }(\mathrm{N}=22)\end{array}$} & $\leq 5$ days & 13 & 59.1 \\
\hline & 1 week & 2 & 9.1 \\
\hline & 2 weeks & 6 & 27.3 \\
\hline & 1 month & 1 & 4.5 \\
\hline \multirow{5}{*}{$\begin{array}{l}\text { Type of antibiotic } \\
\text { used }(\mathrm{N}=22)\end{array}$} & Ceftriaxone & 9 & 40.9 \\
\hline & Ciprofloxacin & 8 & 36.4 \\
\hline & Azythromycin & 3 & 13.6 \\
\hline & Cotrimoxazole & 1 & 4.5 \\
\hline & Amoxycillin & 1 & 4.5 \\
\hline \multirow[t]{2}{*}{ History of UTI (N=137) } & Yes & 25 & 18.3 \\
\hline & No & 112 & 81.7 \\
\hline \multirow{2}{*}{$\begin{array}{l}\text { UTI treatment } \\
\text { facility }(N=25)\end{array}$} & Healthcare facility & 18 & 72 \\
\hline & Pharmacy or drug shops & 7 & 28 \\
\hline \multirow[t]{2}{*}{ History of STD $(\mathrm{N}=137)$} & Yes & 5 & 3.7 \\
\hline & No & 132 & 96.3 \\
\hline \multirow[t]{2}{*}{ STD treatment $(\mathrm{N}=137)$} & Complete & 4 & 80 \\
\hline & Incomplete & 1 & 20 \\
\hline \multirow[t]{2}{*}{ Catheterization $(\mathrm{N}=137)$} & Yes & 1 & 0.7 \\
\hline & No & 136 & 99.3 \\
\hline \multirow{2}{*}{$\begin{array}{l}\text { Other co- } \\
\text { morbid }(\mathrm{N}=137)\end{array}$} & Yes (Hypertension) & 1 & 0.7 \\
\hline & No & 136 & 99.3 \\
\hline
\end{tabular}

Semen analysis, bacteriospermia and ESBL bacteriospermia

All participants $(100 \%)$ had normal semen appearance (color) during semen analysis. Of 137 studied participants, majority of participants had abnormal semen volume (63.5\%): 92.0\% hypospermia and 8.0\% hyperspermia, and poor forward motility of spermatozoa $63.5 \%$. Infertility was observed among $35.0 \%$ of participants of which $64.6 \%$ had oligospermia (Table 2).
Among 137 semen culture, 32.1\% had positive bacteriospermia of which Gram-negative bacteria were predominantly isolated $(86.4 \%)$. K. pneumoniae $(27.3 \%)$ was the most frequently isolated bacteria followed by E. coli $(20.5 \%)$ and Acinetobacter spp. (15.9\%). Out of 38 Gram-negative bacteria, 31.6\% were phenotypically ESBL producers. K. pneumoniae was predominant ESBL producer detected (58.3\%). ESBL blaCTX-M gene was found among 75\% of phenotypically confirmed ESBL producers (Table 2). 
Table 2: Semen analysis, bacteriospermia and ESBL bacteriospermia results

\begin{tabular}{|c|c|c|c|}
\hline Variables & & Frequency (n) & Percentage (\%) \\
\hline \multicolumn{4}{|c|}{ Quality of spermatozoa and semen } \\
\hline \multirow[t]{2}{*}{ Semen appearance $(\mathrm{N}=137)$} & Normal & 137 & 100 \\
\hline & Poor & 0 & 0 \\
\hline \multirow[t]{2}{*}{ Semen PH (N=137) } & Normal $(7.2-8.2)$ & 131 & 95.6 \\
\hline & Increased alkaline $(\geq 9)$ & 6 & 4.4 \\
\hline \multirow[t]{2}{*}{ Semen viscosity $(\mathrm{N}=137)$} & Normal & 55 & 40.1 \\
\hline & Abnormal & 82 & 59.9 \\
\hline \multirow[t]{2}{*}{ Semen volume $(\mathrm{N}=137)$} & Normal & 50 & 36.5 \\
\hline & Abnormal & 87 & 63.5 \\
\hline \multirow[t]{2}{*}{ Abnormal semen volume $(\mathrm{N}=87)$} & Hypospermia & 80 & 92.0 \\
\hline & Hyperspermia & 7 & 8.0 \\
\hline \multirow{2}{*}{$\begin{array}{l}\text { Spermatozoa } \\
\text { morphology }(\mathrm{N}=137)\end{array}$} & Normal & 115 & 83.9 \\
\hline & Abnormal & 22 & 16.1 \\
\hline \multirow[t]{2}{*}{ Spermatozoa motility $(\mathrm{N}=137)$} & Good forward motility & 50 & 36.5 \\
\hline & Poor forward motility & 87 & 63.5 \\
\hline \multicolumn{4}{|c|}{ Quantity of spermatozoa in semen } \\
\hline \multirow[t]{2}{*}{ Male infertility $(\mathrm{N}=137)$} & Normalspermia & 89 & 65.0 \\
\hline & Infertility & 48 & 35.0 \\
\hline \multirow[t]{3}{*}{ Infertility types $(\mathrm{N}=48)$} & Oligospermia $(<20 \mathrm{mil} / \mathrm{ml})$ & 31 & 64.6 \\
\hline & Azoospermia (no sperms) & 16 & 33.3 \\
\hline & Necrospermia (dead) & 1 & 2.1 \\
\hline \multicolumn{4}{|c|}{ Bacteriospermia } \\
\hline \multirow[t]{2}{*}{ Bacteriospermia $(\mathrm{N}=137)$} & Positive & 44 & 32.1 \\
\hline & Negative & 93 & 67.9 \\
\hline \multirow[t]{8}{*}{ Isolated bacteria spp $(\mathrm{N}=44)$} & K. pneumoniae & 12 & 27.3 \\
\hline & E. coli & 9 & 20.5 \\
\hline & Acinetobacter spp & 7 & 15.9 \\
\hline & Enterobacter aerogenes & 3 & 6.8 \\
\hline & Enterococcus faecalis & 3 & 6.8 \\
\hline & K. oxytoca & 3 & 6.8 \\
\hline & $P$. aeruginosa & 3 & 6.8 \\
\hline & Others* & 3 & 6.8 \\
\hline \multicolumn{4}{|c|}{ ESBL Bacteriospermia } \\
\hline \multirow[t]{2}{*}{ ESBL producing GNB $(\mathrm{N}=38)$} & Producers & 12 & 31.6 \\
\hline & None producers & 26 & 64.8 \\
\hline \multirow[t]{4}{*}{ ESBL-GNB species } & K. pneumoniae & 7 & 58.3 \\
\hline & Acinetobacter spp & 3 & 25 \\
\hline & Enterobacter aerogenes & 1 & 8.3 \\
\hline & Pseudomonas aeruginosa & 1 & 8.3 \\
\hline \multirow[t]{2}{*}{ ESBL bla ${ }_{\text {CTX-M3Ggene }(\mathrm{N}=12)}$} & Positive & 9 & 75 \\
\hline & Negative & 3 & 25 \\
\hline
\end{tabular}

The mean age $( \pm S D)$ and mean infertility duration from seeking medical intervention $( \pm S D)$ of the 15 infertile participants with bacteriospermia was $31.5( \pm 8.1)$ years and $2.5( \pm 1.5)$ years, respectively. The majority of in- fertile participants with bacteriospermia had semen hyper-viscosity $(73.3 \%, \mathrm{n}=11)$, hypospermia $(66.7 \%, \mathrm{n}=10)$ and poor forward spermatozoa motility $(66.7 \%, \mathrm{n}=10)$ while $4(26.7 \%)$ participants had no spermatozoa in their semen (Table 3). 
Table 3: Description of 15 infertile participants with bacteriospermia

\begin{tabular}{|l|l|l|l|l|l|l|l|l|l|}
\hline \multirow{2}{*}{$\begin{array}{c}\text { Age } \\
\text { (years) }\end{array}$} & \multirow{2}{*}{$\begin{array}{l}\text { Infertility } \\
\text { duration }\end{array}$} & \multirow{2}{*}{$\begin{array}{l}\text { History } \\
\text { of UTI }\end{array}$} & \multicolumn{5}{|c|}{ Semen analysis } & Bacteria spp & ESBL \\
\hline 25 & 1 & Viscosity & Volume & Morphology & Motility & Remarks & & \\
\hline 20 & 2 & Yes & Abnormal & Reduced & Normal & Normal & Oligospermia & E. faecalis & N/A \\
\hline 33 & 2 & No & Abnormal & Reduced & N/A & N/A & Azoospermia & S. pyogenes & N/A \\
\hline 26 & 1 & No & Abnormal & Increased & Normal & Poor & Oligospermia & E. faecalis & N/A \\
\hline 32 & 3 & No & Abnormal & Normal & Normal & Poor & Oligospermia & E. coli & NEG \\
\hline 26 & 1 & No & Abnormal & Increased & Normal & Poor & Oligospermia & $\begin{array}{l}\text { K. } \\
\text { pneumoniae }\end{array}$ & NEG \\
\hline 45 & 5 & No & Abnormal & Normal & Normal & Poor & Oligospermia & E. coli & NEG \\
\hline 36 & 4 & Normal & Reduced & Normal & Poor & Oligospermia & $\begin{array}{l}\text { K. } \\
\text { pneumoniae }\end{array}$ & POS \\
\hline 35 & 5 & No & Normal & Reduced & N/A & N/A & Azoospermia & $\begin{array}{l}\text { K. } \\
\text { pneumoniae }\end{array}$ & POS \\
\hline 32 & 2 & No & Abnormal & Reduced & Abnormal & Poor & Oligospermia & E. coli & NEG \\
\hline 35 & 3 & Yes & Abnormal & Reduced & Normal & Poor & Oligospermia & $\begin{array}{l}\text { K. } \\
\text { pneumoniae }\end{array}$ & NEG \\
\hline 29 & 1 & No & Normal & Increased & Abnormal & Poor & Oligospermia & $\begin{array}{l}\text { Acinetobacter } \\
\text { spp }\end{array}$ & NEG \\
\hline 49 & 5 & No & Abnormal & Reduced & Normal & Poor & Oligospermia & E. coli & NEG \\
\hline 19 & 1 & No & Normal & Reduced & Normal & Poor & Oligospermia & K. oxytoca & NEG \\
\hline 31 & 2 & No & Abnormal & Reduced & N/A & N/A & Azoospermia & P. aeruginosa & NEG \\
\hline KEY: ID=ldentification number, N/A=Not Applicable, NEG=Negative and POS=Positive & Reduced & N/A & N/A & Azoospermia & $\begin{array}{l}\text { Acinetobacter } \\
\text { spp }\end{array}$ & NEG \\
\hline
\end{tabular}

\section{Antibiotics resistance pattern}

Percentage resistance of Gram-negative bacteria to antibiotics ampicillin, trimethoprim-sulphamethoxazole and amoxycillin-clavulanic acid was $100 \%, 100 \%$ and $92.1 \%$ respectively while ESBL-GNB resistance to ampicillin, trimethoprim-sulphamethoxazole, amoxycillin-clavulanic acid and gentamicin was 100\%, 100\%, 91.7\% and $66.7 \%$. Percentage resistances of Gram-positive bacteria to erythromycin were $66.7 \%$ (Table 4 ).

Table 4: Antibiotics susceptibility patterns of isolated bacteria causing bacteriospermia and ESBL producing GNB

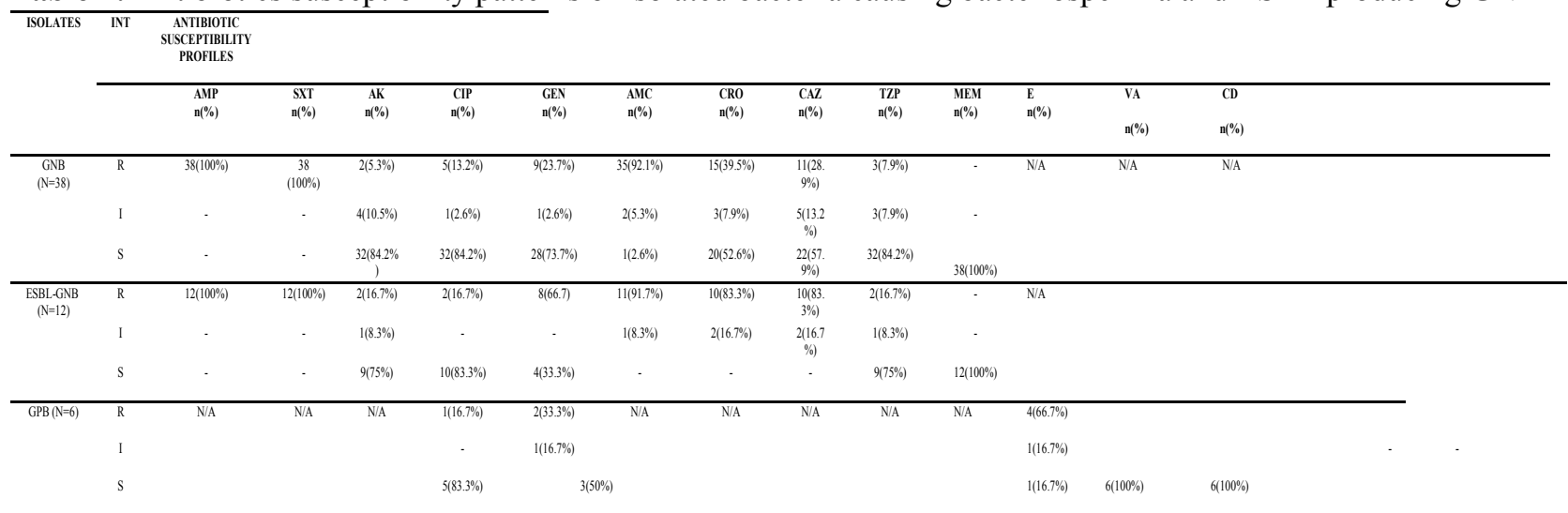

AMP=ampicillin, $\mathrm{SXT}=$ cotrimoxazole, $\mathrm{AK}=$ amikacin, $\mathrm{CIP}=$ ciprofloxacin, $\mathrm{GEN}=$ gentamicin, $\mathrm{AMC}=$ amoxycillin-clavulanic acid, $\mathrm{CRO}=$ ceffriaxone, $\mathrm{CAZ}=$ ceftazidime, $\mathrm{TZP}=$ piperacillin-tazobactam, MEM=meropenem, $\mathrm{E}=$ =rythromycin, $\mathrm{VA}=$ =vancomycin $\mathrm{CD}=$ clindamycin, $\mathrm{INT}=$ interpretation, $\mathrm{R}=$ resistance, $\mathrm{I}=$ intermediate, $\mathrm{S}=$ sensitive and $\mathrm{NA}=$ not applicable

\section{Factors associated with male infertility}

On Chi square analysis, male infertility was significantly associated with: semen hyper-viscosity $(p=0.022)$, abnormal semen volume $(\mathrm{p}=0.015)$, abnormal spermatozoa morphology $(\mathrm{p}<0.001)$ and poor spermatozoa motility $(p<0.001)$.On multivariate regression analysis, male infertility was significantly associated with: antibiotic use (OR (95\%CI): 0.14(0.02-0.85), $\mathrm{p}=0.033)$, abnormal spermatozoa morphology (OR (95\%CI): 14.48(3.1766.05), $\mathrm{p}=0.001)$ and abnormal spermatozoa motility (OR (95\%CI): 0.05(0.01-0.24),p<0.001). Bacteriospermia and ESBL bacteriospermia did not have significant association with infertility on both; univariate and multivariate regression analysis (Table 5). 
Table 5: Factors associated with male infertility among presumptive infertile men

\begin{tabular}{|c|c|c|c|c|c|c|}
\hline \multicolumn{2}{|l|}{ Variables } & \multirow{3}{*}{$\begin{array}{l}\text { Infertility } \\
\mathbf{N}=48, \% \\
2(9.1)\end{array}$} & \multirow{3}{*}{$\begin{array}{l}\text { Chi square } \\
7.7514\end{array}$} & \multirow{3}{*}{$\begin{array}{l}\text { P value } \\
0.005\end{array}$} & \multicolumn{2}{|l|}{ Multivariate } \\
\hline & & & & & \multirow{2}{*}{$\frac{\mathrm{OR}(95 \% \mathrm{CI})}{0.14(0.02-0.85)}$} & \multirow{2}{*}{$\frac{P \text { value }}{0.033}$} \\
\hline Antibiotics use & Yes (22) & & & & & \\
\hline & No (115) & $46(40)$ & & & & \\
\hline \multirow[t]{2}{*}{ History of UTI } & Yes (25) & $3(6.3)$ & 7.1299 & 0.008 & * & $*$ \\
\hline & No (112) & $45(93.7)$ & & & & \\
\hline \multirow[t]{2}{*}{ History of STDs } & Yes (5) & $2(40)$ & 0.0562 & 0.811 & $3.39(0.33-34.82)$ & 0.304 \\
\hline & No (132) & $46(34.8)$ & & & & \\
\hline \multirow[t]{2}{*}{ Semen viscosity } & Hyper (82) & $35(42.6)$ & 5.2469 & 0.022 & $1.18(0.39-3.53)$ & 0.762 \\
\hline & Normal (55) & $13(23.6)$ & & & & \\
\hline \multirow[t]{2}{*}{ Semen volume } & Abnormal (87) & $37(42.5)$ & 5.8790 & 0.015 & $2.85(0.97-8.39)$ & 0.057 \\
\hline & Normal (50) & $11(22)$ & & & & \\
\hline \multirow{2}{*}{$\begin{array}{l}\text { Spermatozoa } \\
\text { morphology }\end{array}$} & Abnormal (22) & $19(39.6)$ & 30.3354 & 0.000 & $14.48(3.17-66.05)$ & 0.001 \\
\hline & Normal (115) & $29(60.4)$ & & & & \\
\hline \multirow{2}{*}{$\begin{array}{l}\text { Spermatozoa } \\
\text { motility }\end{array}$} & Poor (87) & $45(51.7)$ & 29.1655 & 0.000 & $0.05(0.01-0.24)$ & 0.000 \\
\hline & Normal (50) & $3(6.0)$ & & & & \\
\hline \multirow[t]{2}{*}{ Bacteriospermia } & Positive (44) & $15(30.1)$ & 0.0255 & 0.533 & $0.86(0.29-2.59)$ & 0.797 \\
\hline & Negative (93) & $33(35.5)$ & & & & \\
\hline \multirow{2}{*}{$\begin{array}{l}\text { ESBL } \\
\text { Bacteriospermia }\end{array}$} & Positive (12) & $2(16.7)$ & 1.9499 & 0.163 & $0.13(0.01-1.22)$ & 0.073 \\
\hline & Negative (125) & $46(36.8)$ & & & & \\
\hline
\end{tabular}

*had collinearity with previous history of antibiotic use

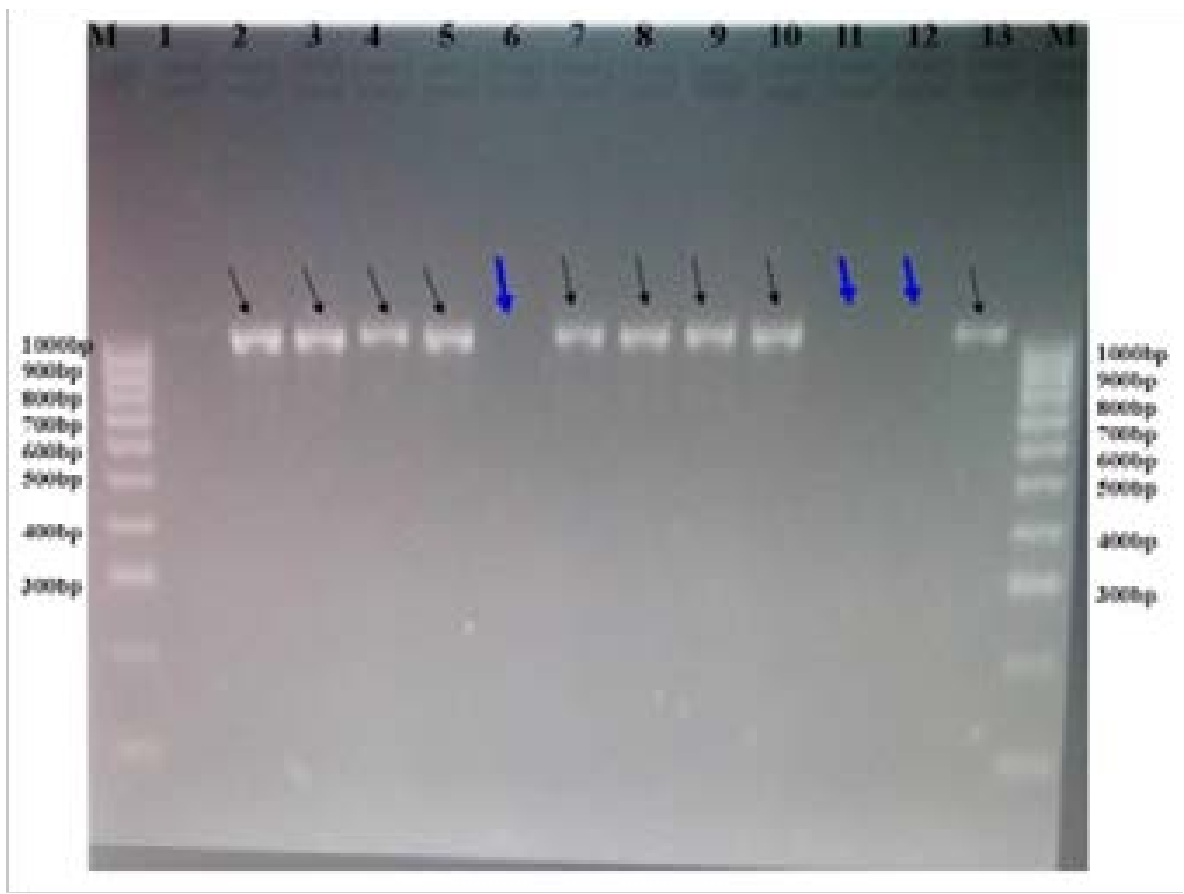

Figure 1: Visualization of PCR products on $2 \%$ agarose gel stained with redsafe. Lane $\mathrm{M}$; ladder marker and lanes 1-13; phenotypic confirmed bacterial isolates. Bacteria with positive bla $a_{\text {CTX-M }}$ genes are shown by black arrows while negative are shown with with thick blue arrows. 


\section{Discussion}

In the current study, male infertility was found among one third of the participants, of which oligospermia was prevalent encountered type followed with azoospermia while one participant had necrospermia as observed previous $^{22}$. Significantly, male infertility was associated with poor forward spermatozoa motility and abnormal spermatozoa morphology. Poor forward spermatozoa motility means that spermatozoa cannot swim properly hence unable to reach the egg for fertilization and abnormal morphology of the spermatozoa means that spermatozoa may be unable to penetrate an egg for fertilization ${ }^{23,24}$. Therefore, the two factors reduce spermatozoa quality and ability of fetilization ${ }^{25}$. These factors may be used as surrogate markers for diagnosis of male infertility ${ }^{14}$.

Neither bacteriospermia nor ESBL bacteriospermia was associated with male infertility as observed elsewhere ${ }^{3,4}$. This might be due to small sample size of this study resulting to wide $95 \% \mathrm{CI}$ and imprecise estimate of the effect therefore results didn't have statistical significance ${ }^{26}$. However, about one third of infertile men had bacteriospermia, suggesting that bacteria might have adverse impact on spermatozoa quantity and/or quality. This is further supported by the fact that history of antibiotic use was protective factor of male infertility. Therefore, there is a need of infectology to be part of infertility diagnosis and management among presumptive infertile men.

Gram-negative bacteria, specifically K. pneumoniae, E. coli and Acinetobacter spp. were prevalently isolated in this study as previously reported from other studies ${ }^{22,27}$. This is contrary to a study3 which reported that, Gram-positive bacteria, specifically Enterococcus faecalis and Staphylococcus aureus were predominantly isolates causing bacteriospermia. This difference may be due to overall increase trend of multi resistant gram negative infections as the commonest cause of bacterial infections, in the current study's setting Gram-negative bacteria are the most leading causative agents of bacterial infections ${ }^{10}$.

About one third of the Gram-negative bacteria were found to be ESBL producers with three quarters of phenotypic ESBL producers carrying blaCTX-M gene. The other quarter might be carrying other CTX-M groups and/or other ESBL families (SHV and TEM) as previously observed $^{28}$. It should be noted previously studies ${ }^{21,29,30}$ have found blaCTX-M-15 which is a member of group 1 to be the commonest allele $(>75 \%)$ in the majority of ESBL producers in Tanzania. The observation of about 30\% of Gram-negative bacteria from semen culture to carry ESBL genes is significantly higher than what has been observed in other studies ${ }^{31,32}$. This could be due to high ESBL carriage in our setting due to overuse of antibiotics $^{29,33}$. In this study it was observed that $16.1 \%$ of participants used antibiotics mainly cephalosporins (40.9\%) without prescriptions. As previously observed, in the current study, resistance to non -beta lactam antibiotics was very high among ESBL- producers ${ }^{33}$. The observation is worrisome as treatment options for ESBL producing bacteria are expensive and most of the time not available in most health facilities in developing countries.

\section{Conclusion}

The magnitude of bacteriospermia and ESBL bacteriospermia is high among presumptive infertile men. We recommend that, infectology should be part of diagnosis and management of male infertility.

\section{Limitations of the study}

Due to limited funds, this study neither characterized other ESBL families (SHV and TEM) nor other CTX-M groups. Furthermore, we did not investigate other pathogens such as fungi and viruses which could have adverse impact on the quality and/or quantity of spermatozoa in semen of infertile men. Another limitation is, we lack data schistosomiasis screening which is endemic in this study setting and reported elsewhere to be associated with male infertility.

\section{Competing interests}

None declared.

\section{Authors' contributions}

VS, JI, FC, MFM and SEM conceived and designed this study; YM and ALH collected study data; VS, YM and ALH participated in laboratory procedures; VS, JI, FC, MFM and SEM participated in data analysis; VS wrote the first draft of manuscript; all authors critically revised and approved the final draft of manuscript.

\section{Acknowledgment}

The authors wish to thank laboratory technicians and scientists from Central Pathology Laboratory, department of Histopathology at Bugando Medical Centre for technical assistance and Mr. Bernard Okamo for optimization of PCR for characterizations of CTX-M gene. 


\section{References}

1. Diemer T, Huwe P, Ludwig M, Hauck E, Weidner W. Urogenital infection and sperm motility. Andrologia. 2003;35(5):283-287.

2. Ochsendorf F. Sexually transmitted infections: impact on male fertility. Andrologia. 2008;40(2):72-75.

3. Vilvanathan S, Kandasamy B, Jayachandran AL, et al. Bacteriospermia and its impact on basic semen parameters among infertile men. Interdisciplinary Perspectives on Infectious Diseases. 2016;2016.

4. Golshani M, Taheri S, Eslami G, Rahbar AS, Fallah F, Goudarzi H. Genital tract infection in asymptomatic infertile men and its effect on semen quality. Iranian Journal of Public Health. 2006;35(3):81-84.

5. Bukharin O, Kuz'min M, Ivanov I. The role of the microbial factor in the pathogenesis of male infertility. Zhurnal Mikrobiologii, Epidemiologii, I Immunobiologii. 2000(2):106-110.

6. Heffernan H, Pope C, Carter P. Identification of extended-spectrum $\beta$-lactamase types, plasmid mediated AmpC $\beta$-lactamases and strains among urinary Escherichia coli and Klebsiella in New Zealand in 2006. Communicable Disease Group, ESR. FW07103. 2007.

7. Iredell J, Brown J, Tagg K. Antibiotic resistance in Enterobacteriaceae: mechanisms and clinical implications. BMJ. 2016;352:h6420.

8. Mshana SE, Hain T, Domann E, Lyamuya EF, Chakraborty T, Imirzalioglu C. Predominance of Klebsiella pneumoniae ST14 carrying CTX-M-15 causing neonatal sepsis in Tanzania. BMC Infectious Diseases. 2013;13(1):466.

9. Marando R, Seni J, Mirambo MM, et al. Predictors of the extended-spectrum-beta lactamases producing Enterobacteriaceae neonatal sepsis at a tertiary Hospital, Tanzania. International Journal of Medical Microbiology. 2018. 10. Kayange N, Kamugisha E, Mwizamholya DL, Jeremiah S, Mshana SE. Predictors of positive blood culture and deaths among neonates with suspected neonatal sepsis in a tertiary hospital, Mwanza-Tanzania. BMC Pediatrics. 2010;10(1):39.

11. Seni J, Sweya E, Mabewa A, Mshana SE, Gilyoma JM. Comparison of antimicrobial resistance patterns of ESBL and non ESBL bacterial isolates among patients with secondary peritonitis at Bugando Medical Centre, MwanzaTanzania. BMC Emergency Medicine. 2016;16(1):41.

12. World Health Organization D. WHO laboratory manual for the Examination and processing of human sperm. World Health Organiz. 2010.
13. WHO Laboratory Manual for the Examination of Human Semen and Semen-Cervical Mucus Interlaction [press release]. Cambridge Cambridge University Press1999.

14. Cooper TG, Noonan E, Von Eckardstein S, et al. World Health Organization reference values for human semen characteristics. Human Reproduction Update. 2010;16(3):231-245.

15. Plachot M, Belaisch-Allart J, Mayenga J-M, Chouraqui A, Tesquier L, Serkine AM. Outcome of conventional IVF and ICSI on sibling oocytes in mild male factor infertility. Human Reproduction. 2002;17(2):362-369.

16. Koneman EW, Allen SD, Janda W, Schreckenberger P, Winn W. Diagnostic microbiology. The nonfermentative gram-negative bacilli. Philedelphia: Lippincott-Raven Publishers. 1997:253-320.

17. Wayne P. Clinical and Laboratory Standards Institute: Performance standards for antimicrobial susceptibility testing: 20th informational supplement. CLSI document M100-S20. 2010.

18. Drieux L, Brossier F, Sougakoff W, Jarlier V. Phenotypic detection of extended-spectrum $\beta$-lactamase production in Enterobacteriaceae: review and bench guide. Clinical Microbiology and Infection. 2008;14:90-103.

19. Livermore DM. beta-Lactamases in laboratory and clinical resistance. Clinical Microbiology Reviews. 1995;8(4):557-584.

20. Dashti AA, Jadaon MM, Abdulsamad AM, Dashti HM. Heat treatment of bacteria: A simple method of DNA extraction for molecular techniques. Kuwait Med J. 2009;41(2):117-122.

21. Mshana SE, Imirzalioglu C, Hossain $H$, Hain $T$, Domann E, Chakraborty T. Conjugative IncFI plasmids carrying CTX-M-15 among Escherichia coli ESBL producing isolates at a University hospital in Germany. BMC Infectious Diseases. 2009;9(1):97.

22. Ahmad S, Wasim S, Tiwari N, Verma V, Gupta N, Mishra N. Evaluation of Bacteriospermia as Etiology for Oligospermia: An Analysis. International Journal of Scientific Study. 2016;4(2):194-197.

23. Kristiansen S. The Causes of Low Sperm Motility and How It Can Be Treated. 2015; https://www.infertilityivfhouston.com/blog/2015/06/16/the-causes-of-lowsperm-159391. Accessed 02 September 2018.

24. Madormo C. How Does Sperm Morphology Affect Fertility? 2017; https://www.healthline.com/health/ sperm-morphology. Accessed 02 September 2018.

25. Fisch H. Declining worldwide sperm counts: disprov- 
ing a myth. Urologic Clinics of North America. 2008;35(2):137146.

26. Hackshaw A. Small studies: strengths and limitations. Eur Respiratory Soc; 2008.

27. Bhatt C, Mishra S, Bhatt A, Lakhey M. Bacterial pathogens in semen culture and their antibiotic susceptibility pattern in vitro. Int J Biomed Res. 2015;6(11):909-914. 28. Moremi N, Claus H, Vogel U, Mshana SE. Faecal carriage of CTX-M extended-spectrum beta-lactamase-producing Enterobacteriaceae among street children dwelling in Mwanza city, Tanzania. PloS One. 2017;12(9):e0184592. 29. Mshana SE, Hain T, Domann E, Lyamuya EF, Chakraborty T, Imirzalioglu C. Predominance of Klebsiella pneumoniaeST14 carrying CTX-M-15 causing neonatal sepsis in Tanzania. BMC Infectious Diseases. 2013;13(1):466.

30. Moremi N, Manda EV, Falgenhauer L, et al. Predomi- nance of CTX-M-15 among ESBL producers from environment and fish gut from the shores of Lake Victoria in Mwanza, Tanzania. Frontiers in Microbiology. 2016;7.

31. Ibrahim ME, Bilal NE, Magzoub MA, Hamid ME. Prevalence of extended-spectrum $\beta$-lactamases-producing Escherichia coli from Hospitals in Khartoum State, Sudan. Oman Medical Journal. 2013;28(2):116.

32. Adeyankinnu FA, Motayo BO, Akinduti A, et al. A multicenter study of beta-lactamase resistant Escherichia coli and Klebsiella pneumoniae reveals high level chromosome mediated extended spectrum $\beta$ lactamase resistance in Ogun State, Nigeria. Interdisciplinary Perspectives on Infectious Diseases. 2014;2014.

33. Marwa KJ, Mushi MF, Konje E, Alele PE, Kidola J, Mirambo MM. Resistance to cotrimoxazole and other antimicrobials among isolates from HIV/AIDS and NonHIV/AIDS patients at bugando medical centre, Mwanza, Tanzania. AIDS Research and Treatment. 2015;2015. 